\title{
New Approach for Improvement of Secondary Ion Mass Spectrometry Profile Analysis
}

\author{
M'hamed Boulakroune ${ }^{1,2 *}$, Ahmed El OuAlKADi ${ }^{3}$, Djamel BenAtia ${ }^{2}$, and Tahar KeZAi ${ }^{3}$ \\ ${ }^{1}$ Telecom Laboratory, Electrical Engineering Department, Bt Stevin, Université Catholique de Louvain, \\ Place de Levant 2, B-1348, Louvain-la-Neuve, Belgium \\ ${ }^{2}$ Electronics Department, Faculty of Engineer Sciences, Université Colonel Hadj-Lakhdar de Batna, Batna 05000, Algeria \\ ${ }^{3}$ Microelectronics Laboratory, Electrical Engineering Department, Université Catholique de Louvain, \\ Bt Maxwell, place de Levant 3, B-1348, Louvain-la-Neuve, Belgium
}

(Received April 3, 2007; accepted July 30, 2007; published online November 6, 2007)

In this paper, we describe the improvement of secondary ion mass spectrometry (SIMS) profile analysis by a new approach based on partial deconvolution combined with scale-frequency shrinkage. The SIMS profiles are obtained by analysis of the delta layers of boron doped silicon in a silicon matrix, analyzed using Cameca-Ims $6 f$ at oblique incidence. These profiles can be approximated closely by exponential-like tail distributions with decay length, which characterizes the collisional mixing effect. The partial deconvolution removes the residual ion mixing effect. The contributions of high-frequency noise are removed by shrinkage to a great extent of the profiles. It is shown that this approach leads to a marked improvement in depth resolution without producing artifacts and aberrations caused principally by noise. Furthermore, it is shown that the asymmetry of the delta layers, caused by the collisional mixing effect, is completely removed, the decay length is decreased by a factor of 4 compared with that before deconvolution. [DOI: 10.1143/JJAP.46.7441]

KEYWORDS: SIMS, multilayers, in-depth resolution, scale-frequency shrinkage, partial deconvolution

\section{Introduction}

Secondary ion mass spectrometry (SIMS) is widely used in the semiconductor industry, particularly for the development of advanced integrated circuits and devices. It is a versatile technique that can be applied to any problem requiring in-depth analysis. ${ }^{1)}$

Nowadays, device size is scaled down, which requires considerable effort on the improvement of SIMS apparatuses, in order to realize high sensitivity, high lateral resolution and in-depth resolution. ${ }^{1,2)}$ However, depth resolution is governed by several phenomena inherent to the action of the primary ion in the substrate and their incorporation in the matrix. The main physical mechanism responsible for the degradation of depth resolution is collisional mixing. This results from the collision cascades following the loss of the primary ion energy in the target, and greatly limits the accuracy of depth profile determinations at nanometer depth resolutions. This phenomenon cannot be completely removed, but it can be reduced by lowering the primary ion energies. ${ }^{3)}$ In addition to this physical phenomenon, noise is an instrumental parameter that cannot be eliminated by the improvement of operative conditions. Moreover, the development of more sophisticated instrumentation might enhance the skills of SIMS operators. ${ }^{4)}$ Fortunately, the experimental depth resolution, as well as the shape of the SIMS profile, can be improved by deconvolution. Nevertheless, recent years have seen the development of several deconvolution algorithms that show an improvement in depth resolution without taking into account noise effects. ${ }^{2-6)}$ Indeed, the consequence of noise in numerical processing is the generation of artifacts and oscillations that limit the accuracy of results and their interpretations. Moreover, none of these algorithms have been used widely because the calculations are typically complicated and time-consuming. ${ }^{1)}$ These algorithms require specific and detailed knowledge of response functions from the measurements of $\delta$-doped samples, which are often not available. The obtained results using almost of these

*E-mail address: Boulakroune@tele.ucl.ac.be algorithms show aberrations and negatives concentrations, which are not physically acceptable. . $^{1,3,5,6)}$

In this paper, we propose a new deconvolution algorithm based on partial deconvolution ${ }^{1,5)}$ combined with an algorithm widely used in speech processing for noise compensation, which is called scale-frequency shrinkage. ${ }^{8)}$ Partial deconvoution is used to remove the collisional mixing effect. Shrinkage is used to remove artifacts and oscillations from the analyzed results to provide more accurate measurements of depth profiles. The case of multilayer boron-doped silicon, analyzed using Cameca-Ims $6 f$ at oblique incidence, is considered. The obtained results using this approach are presented and discussed.

\section{Principle of Proposed Algorithm}

The two principle mechanisms responsible for the broadening of the shape and depth resolution of SIMS profiles are collisional mixing and noise. Indeed, it is important to eliminate these phenomena to retrieve the shape and spatial resolution of SIMS profiles. To improve the quality of SIMS profiles, we propose a new algorithm based on scalefrequency shrinkage combined with partial deconvolution. This algorithm depends only on local depth information and does not require detailed knowledge of the entire profile (Fig. 1).

\subsection{Scale-frequency shrinkage}

In the signal processing theory, denoising is a major issue, which is widely reported in the literature. ${ }^{8,9)}$ If the observed noisy signal is $y_{n}(z)=y(z)+n(z)$ (where $y$ is the perfect signal and $n$ is the noise added independently to the perfect signal) the main question is how to obtain the estimated $\hat{y}(z)$ of the perfect signal $y(z)$. In probabilistic terms, $y$ and $n$ are regarded as achievements of the random variables $Y$ and $N$. According to the used model for these random variables, several denoising methods were proposed. ${ }^{8-11)}$ In this paper, scale-frequency shrinkage has been used. This algorithm is largely used in signal wavelet transform. ${ }^{8)}$

The soft shrinkage corresponds to the maximum a posteriori (MAP) estimation of $y$, with the assumption that $N$ is 


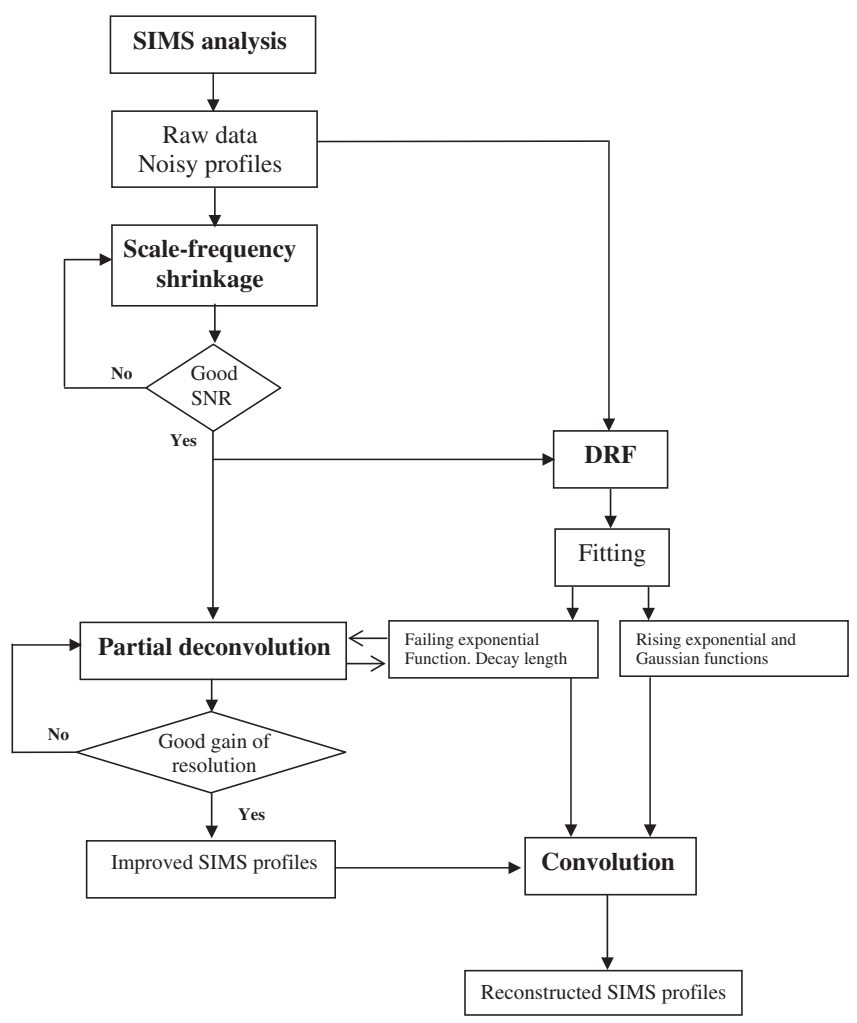

Fig. 1. Algorithm of proposed approach.

a white-Gaussian noise, which is a type of noise in SIMS analysis. The estimation is carried out using soft shrinkage as follows:

If

$$
\left|y_{n}(z, f)\right|>\beta(f),
$$

then,

$$
\hat{y}(z, f)=\frac{y_{n}(z, f)}{\left|y_{n}(z, f)\right|}\left(\left|y_{n}(z, f)-\beta(f)\right|\right)
$$

else,

$$
\hat{y}(z, f)=0,
$$

where, $f$ is the frequency, $z$ is the scale, and $B(f)$ is the threshold of shrinkage defined by

$$
\beta(f)=\alpha \frac{\sigma_{n}^{2}(f)}{\sigma_{y}^{2}(f)},
$$

where $\alpha$ is a parameter that controls the amount of removed noise. $\sigma_{n}$ and $\sigma_{y}$ are, respectively, the standard deviation of the noise and the perfect signal. The estimation of $\sigma_{n}^{2}(f)$ and $\sigma_{y}^{2}(f)$ is carried out by estimating power spectral density, considering the value of signal-to-noise ratio (SNR) that gives the best result. By using this procedure, the noise can be smoothed out; thus, artifacts and oscillations resulting from deconvolution can be avoided.

\subsection{Partial deconvolution}

Yang et al. ${ }^{1,5)}$ have identified the artificial tailing observed in SIMS profiles as primarily caused by the residual collisional mixing effect, characterized by an exponential decay of the peak concentration. The decay length $\lambda_{\mathrm{d}}$ generally depends on the tracer element and matrix, primary

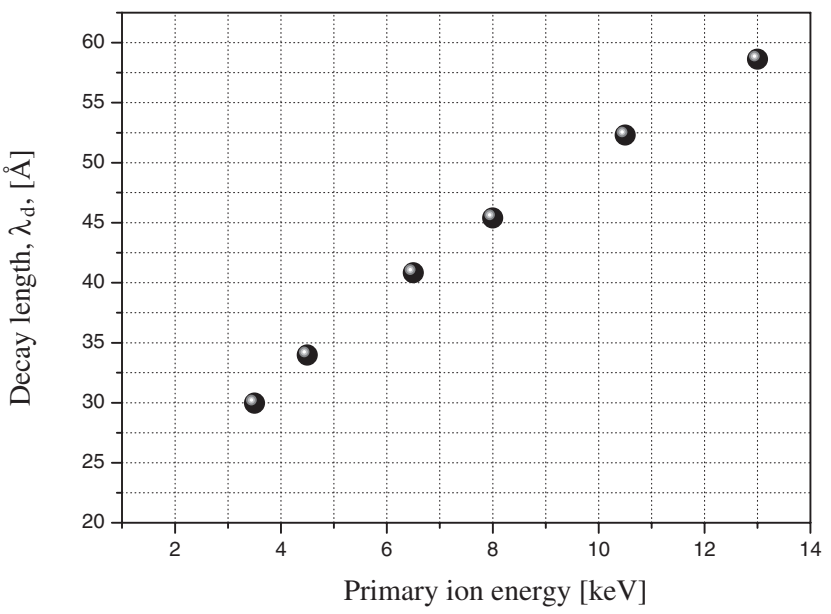

Fig. 2. Evolution of exponential decay $\left(\lambda_{d}\right)$ versus primary ion energy.

ion beam characteristics, and particularly impact ion energy (Fig. 2).

To remove the residual ion mixing effect, Yang et al. proposed an algorithm based on partial deconvolution. The SIMS response function $h\left(z-z^{\prime}\right)$ is the normalized response at any given depth $(z)$ of a $\delta$-doped layer at $\left(z^{\prime}\right)$ depth, when the analysis process is linear and invariant. A real concentration $x(z)$ will result in a measured profile $y_{n}(z)$ defined by

$$
y_{n}(z)=\int_{-\infty}^{+\infty} x\left(z^{\prime}\right) h\left(z-z^{\prime}\right) d z^{\prime}+n(z),
$$

where $n(z)$ is the noise that is added independently to the perfect measured signal $y(z)$. After denoising, the estimation of the denoisy signal $\hat{y}(z)$ is expressed by

$$
\hat{y}(z)=\int_{-\infty}^{+\infty} x\left(z^{\prime}\right) h\left(z-z^{\prime}\right) d z^{\prime} .
$$

The collisional mixing effect is an exponential decay function that is modeled by

$$
\begin{cases}\operatorname{dec}\left(z-z^{\prime}\right)=\frac{1}{\lambda_{\mathrm{d}}} \exp \left(-\frac{z-z^{\prime}}{\lambda_{\mathrm{d}}}\right) & z>z^{\prime} ; \\ \operatorname{dec}\left(z-z^{\prime}\right)=0 & z<z^{\prime} .\end{cases}
$$

The general SIMS response function $h(z)$ (depth resolution function, DRF) can be expressed as a convolution of the collisional mixing function $\left[\operatorname{dec}\left(z-z^{\prime}\right)\right]$ with different convolution functions $\left[g\left(z-z^{\prime}\right)\right]$;

$$
h\left(z-z^{\prime}\right)=\int_{-\infty}^{+\infty} \operatorname{dec}(\tau) \cdot g\left(z-z^{\prime}-\tau\right) d \tau .
$$

The differentiation of both sides of eq. (5) with respect to $z$, then substitution into eqs. (6) and (7) yields

$$
\int_{-\infty}^{+\infty} x\left(z^{\prime}\right) \cdot g\left(z-z^{\prime}\right) d z^{\prime}=\hat{y}(z)+\lambda_{\mathrm{d}} \frac{\partial \hat{y}(z)}{\partial z} .
$$

Considering the approximation at the zeroth order (i.e., assuming $g(z)$ as a delta function that can be generalized in the next paragraph) then

$$
x(z) \approx \hat{y}(z)+\lambda_{\mathrm{d}} \frac{\partial \hat{y}(z)}{\partial z} .
$$

From this relation, the deconvolved signal $[x(z)]$ depends only on the measured signal $[\hat{y}(z)]$ and its derivate multiplied 
by the exponential decay length. It does not require specific and detailed knowledge of DRF, which is often not available.

\subsection{Reconstruction of SIMS profiles}

To generalize and validate the proposed algorithm, it is important to reconstruct SIMS profiles by simulation. The simulation procedure is as follows: Let us consider $y_{1}$ and $y_{2}$ to be the two experimental measurements of profile $x$ analyzed respectively at two different energies, $E_{1}$ and $E_{2}$. By definition,

$$
\begin{aligned}
& \hat{y}_{1}=h\left(z, \lambda_{\mathrm{u} 1}, \sigma_{\mathrm{g} 1}, \lambda_{\mathrm{d} 1}\right) \otimes x(z) ; \\
& \hat{y}_{2}=h\left(z, \lambda_{\mathrm{u} 2}, \sigma_{\mathrm{g} 2}, \lambda_{\mathrm{d} 2}\right) \otimes x(z) .
\end{aligned}
$$

By substituting the two equations of eq. (10), we obtain

$$
\hat{y}_{2}=h\left(z, \lambda_{\mathrm{u} 2}, \sigma_{\mathrm{g} 2}, \lambda_{\mathrm{d} 2}\right) \otimes\left(\hat{y}_{1} \Theta h\left(z, \lambda_{\mathrm{u} 1}, \sigma_{\mathrm{g} 1}, \lambda_{\mathrm{d} 1}\right)\right),
$$

where operators $\otimes$ and $\Theta$ are, respectively, the convolution and deconvolution operators. $\lambda_{\mathrm{u}}$ and $\sigma_{\mathrm{g}}$, are, respectively, the rising exponential decay and standard deviation of the Gaussain function.

Since the partial deconvolution is used, then we have $\tilde{x}=$ $x \otimes g\left(\lambda_{\mathrm{u} 1}, \sigma_{\mathrm{g} 1}\right)$ rather than $x$. The function $g\left(\lambda_{\mathrm{u}}, \sigma_{\mathrm{g}}\right)$, which has been considered in $\$ 2.2$ as Dirac function [approximation at the zeroth order, eq. (7)], contains the Gaussian and exponential rising contributions. This function is the result of the convolution of a rising exponential function (of the parameter $\lambda_{\mathrm{u}}$ ) with a Gaussian function (of parameter $\sigma_{\mathrm{g}}$ ). The relation to be verified then is

$$
\hat{y}_{2}=h\left(z, \lambda_{\mathrm{u} 2}, \sigma_{\mathrm{g} 2}, \lambda_{\mathrm{d} 2}\right) \otimes\left(\tilde{x}(z) \Theta g\left(z, \lambda_{\mathrm{u} 1}, \sigma_{\mathrm{g} 1}\right)\right) ;
$$

then,

$$
\hat{y}_{2}=h\left(z, \sqrt{\sigma_{\mathrm{g} 2}^{2}-\sigma_{\mathrm{g} 1}^{2}}, \lambda_{\mathrm{d} 2}\right) \otimes \tilde{x}(z) .
$$

From the deconvolved profiles, this simulation procedure has been applied to reconstruct SIMS profiles analyzed at different energies.

\section{Results and Discussion}

Samples of boron doped in silicon are multilayers analyzed using Cameca-Ims $6 f$ magnetic sector instrument, corresponding to $3.5 \mathrm{keV} / \mathrm{O}^{+}{ }_{2}$ primary beam $\left(49.0^{\circ}\right.$ incidence) and $6.5 \mathrm{keV} / \mathrm{O}^{+}{ }_{2}$ primary beam (40.3 ${ }^{\circ}$ incidence). The SIMS profiles are shown in Figs. 3 and 4 . The total sputter depth was determined from the crater measurements.

The distorted tail shape observed in the boron profile is due to a significantly larger ion mixing effect. This is characterized by the decay length $\lambda_{\mathrm{d}}$, which is the most important and sensitive parameter. It is equal to $30 \AA$ for the profile analyzed at $3.5 \mathrm{keV}$ (Fig. 3) and to $40.7 \AA$ for the profile analyzed at $6.5 \mathrm{keV}$ (Fig. 4). This parameter has been determined by fitting the exponential decay function to the decay tail. At high frequencies, the noise affects the low levels of signals [this appears clearly in the logarithmic scale of Figs. 3(b) and 4(b)].

By using the proposed algorithm, the results are quite satisfactory suggesting that this approach is indeed selfconsistent. A clear improvement in the contrast is observed; the delta layers are more separated. The shape of the results is symmetrical for all layers, indicating that the exponential features caused by SIMS analysis are removed. The new

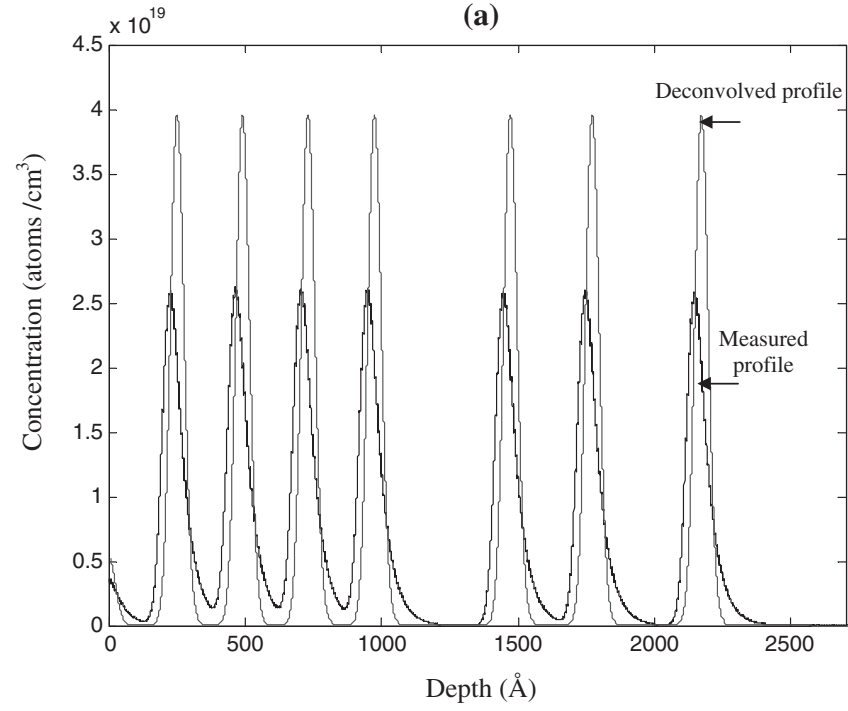

(b)

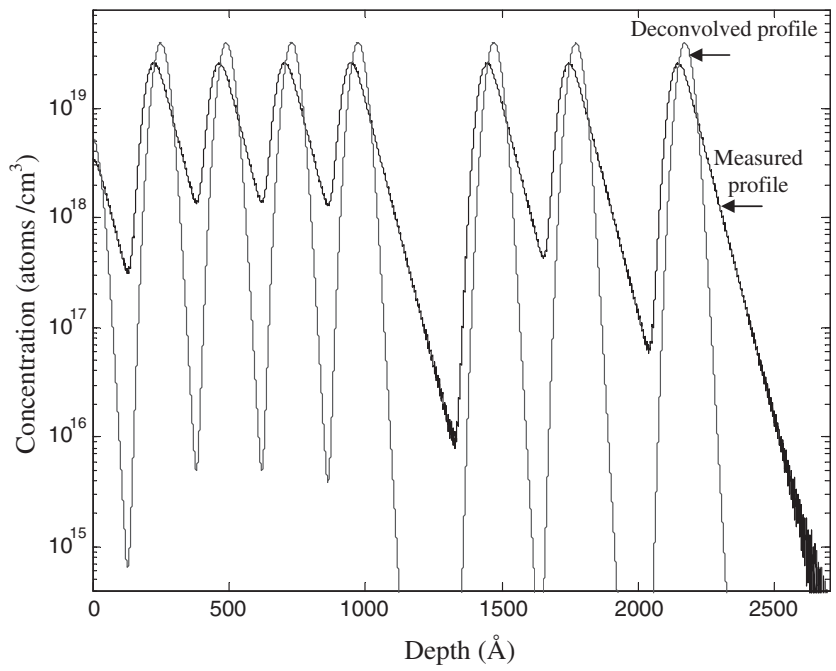

Fig. 3. Deconvolution results of SIMS profile measured at $3.5 \mathrm{keV} / \mathrm{O}_{2}{ }^{+}$ $\left(49.0^{\circ}\right)$ : (a) linear scale and (b) logarithmic scale.

values of $\lambda_{\mathrm{d}}$ are, respectively, 6.7 and $9 \AA$ for the profiles corresponding to 3.5 (Fig. 3) and $6.5 \mathrm{keV}$ (Fig. 4). These values give a gain approximately equal to 4.5 . The in-depth resolution is characterized by the full width at half maximum (FWHM) of the deconvolved delta layers. The in-depth resolutions are $58.65 \AA$ for the profile corresponding to $3.5 \mathrm{keV}$ (Fig. 3) and $91.46 \AA$ for the profile corresponding to $6.5 \mathrm{keV}$ (Fig. 4) thus, the FWHMs of the measured deltas of each profile are, respectively, 118.03 and $174.98 \AA$, which give a gain of in-depth resolution of 2 . The dynamic range is improved by a factor of 1.5 for both profiles [clearly observed in the linear scale of Figs. 3(a) and 4(a)]. The heights of the delta layers have been corrected so that all the layers have approximately the same height, as modified by SIMS analysis. Moreover, the obtained results show the conservation of the rising part and round form of the deconvolved peaks due to the absence of the correction of the rising exponential and the Gaussian part of the depth resolution function.

Indeed, partial deconvolution is sensitive to noise, because it is not regularized; the numerical derivation of the noisy 


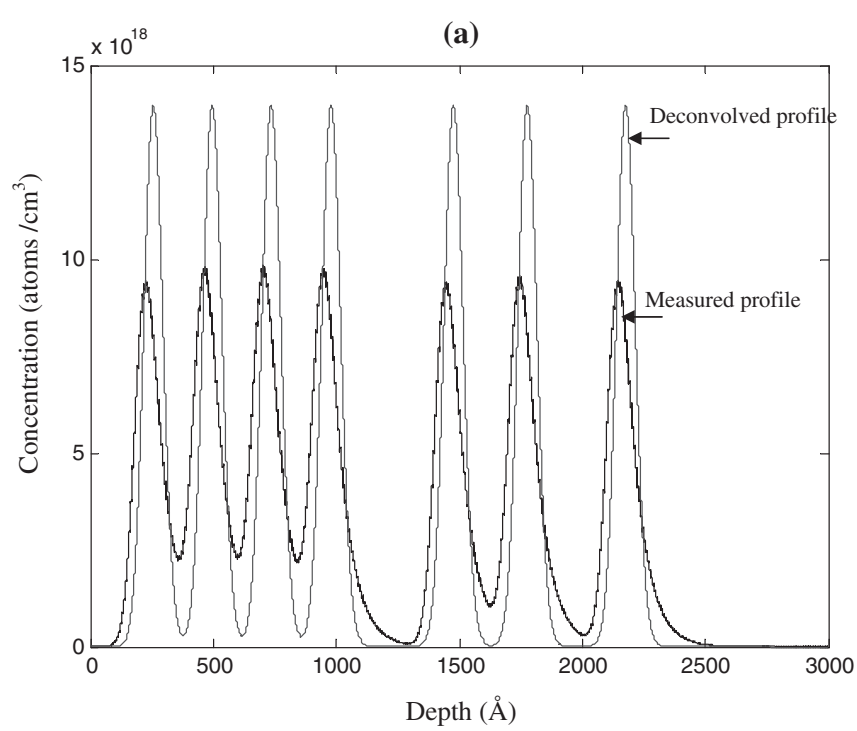

(b)

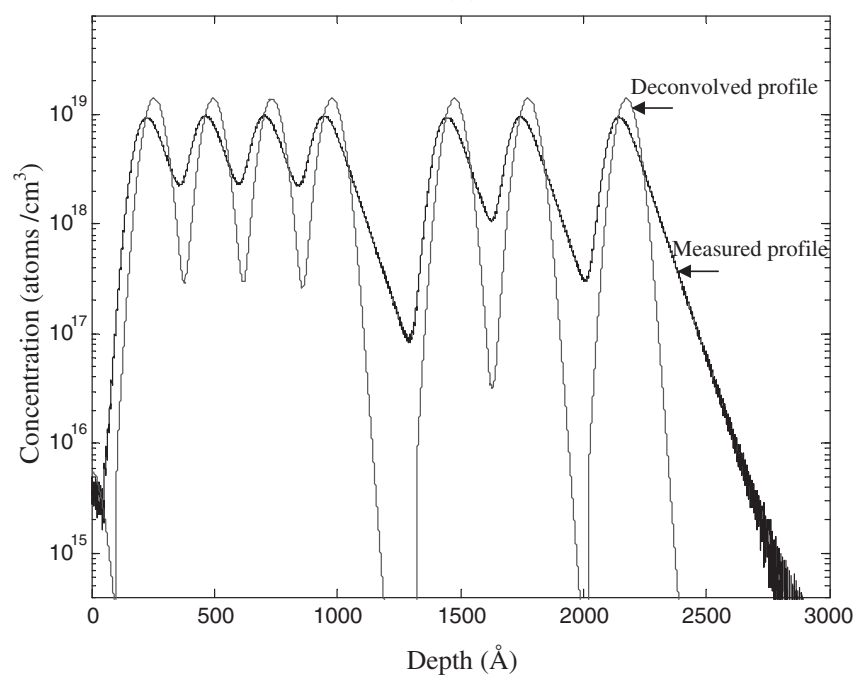

Fig. 4. Deconvolution results of SIMS profile measured at $6.5 \mathrm{keV} / \mathrm{O}_{2}{ }^{+}$ $\left(40.3^{\circ}\right)$ : (a) linear scale and (b) logarithmic scale.

data generates values potentially aberrant and the final result can contain negative values, oscillations and artifacts. For this reason, it was essential to use a procedure of denoising before any numerical processing. By using scale-frequency shrinkage, there are no artifacts and oscillations (which are the consequences of noise) at the end of the deconvolved peaks; fortunately the noise is removed before deconvolution. The estimated SNR values are equal to 40 and $35 \mathrm{~dB}$, for the profiles measured at 3.5 and $6.5 \mathrm{keV}$, respectively. The accuracy of this algorithm depends on the precision of the SNR obtained from the measurement, and the accuracy and applicability of the fitting parameters of the depth resolution function.

On the other hand, the first-order moment of $h(z)$ is equal to $\lambda_{\mathrm{d}}-\lambda_{\mathrm{u}} \approx \lambda_{\mathrm{d}}\left(\lambda_{\mathrm{u}}\right.$ is practically negligible in the DRF; it is approximately equal within 4 to $5 \AA$ ). Thus, a profile treated by using this procedure must be shifted towards the righthand side, ${ }^{1,12)}$ except if one allots to the phenomena a conservative character of the average position of the profiles. In this study, it is supposed, implicitly, that the depth resolution function is invariant; this procedure, like any

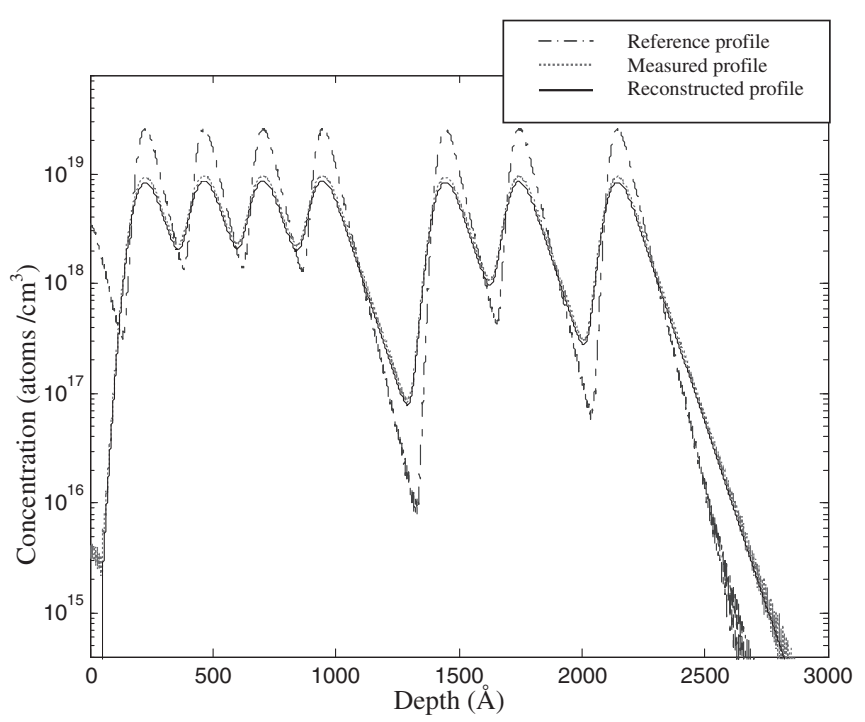

Fig. 5. Reconstruction of SIMS profile measured at $6.5 \mathrm{keV}$ from reference profile measured at $3.5 \mathrm{keV}$. Plain line: simulated profile; doted line: measured profile; noisy line: reference profile.

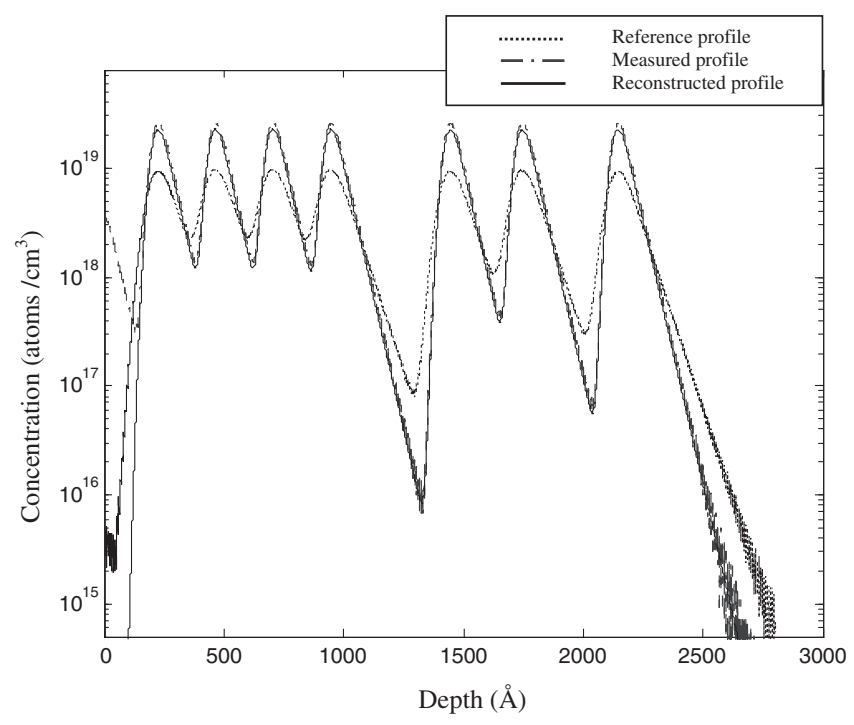

Fig. 6. Reconstruction of SIMS profile measured at $3.5 \mathrm{keV}$ from reference profile measured at $6.5 \mathrm{keV}$. Noisy line: measured profile; plain line: reconstructed profile; dotted line: reference profile.

other procedure of deconvolution based on this assumption, can be applied only in the part of the profile that corresponds to the steady state of the analysis.

Indeed, this deconvolution is not complete because the true DRF is not reduced to a simple exponential. However, it is partial deconvolution in a sense that improves the result without introducing additional artifacts. Note that the failing exponential deformation of the profiles constitutes the most significant part of deformation as the primary ion energy increases. Moreover, a profile convolved by the true DRF, then partially deconvolved, is equivalent to the original profile convolved by the Gaussian and rising exponential functions.

Figures 5 and 6 (in logarithmic scale) show the reconstructed and the measured profiles. The reference profile $\left(y_{1}\right)$ (measured at $3.5 \mathrm{keV}$ ) is partially deconvolved using eq. (9), and then the profile (measured at $6.5 \mathrm{keV}$ ) is reconstructed 
from the deconvolved reference using eq. (13) (Fig. 5). By using the same process and from the reference profile $\left(y_{2}\right)$ (measured at $6.5 \mathrm{keV}$ ), the profile $\left(y_{1}\right)$ (measured at $3.5 \mathrm{keV}$ ) is then reconstructed (Fig. 6).

A good agreement has been obtained between the reconstructed and measured profiles over most of the profile depths. The simulation and measured profiles are practically superimposed. The reconstructed profiles cannot be distinguished from the measured profiles.

\section{Conclusions}

The aim of using deconvolution in SIMS profiles is to retrieve the shape and to improve depth resolution broadened by the analysis. A more appropriate and flexible method is the restitution of profiles taking into account the main mechanisms governing the analysis. These mechanisms are collisional mixing and noise. By using the proposed algorithm, these phenomena are removed leading to smooth and accurate results. It is shown that the shape of all delta layers is partially retrieved in a very satisfactory manner. The exponential behavior, caused by the collisional mixing, has been completely removed and the heights of the delta layers have been corrected. The distance between peaks and their symmetry have been restored and in-depth resolution has been improved by a factor close to 2 . Moreover, the proposed algorithm allows the removal of artifacts and oscillations at the end of the delta peaks, which are smoothed by denoising. By reconstructing the profiles, the obtained results are validated and they show good agreement between the simulation and measured profiles.

1) M. H. Yang, Y. Li, C. Hitzman, and R. Odom: Mater. Res. Soc. Symp. Proc. 669 (2001) J4.16.

2) Y. Kawashima, T. Ide, S. Aoyagi, and M. Kudo: Appl. Surf. Sci. 231232 (2004) 800.

3) B. Fares, B. Gautier, J. C. Dupuy, G. Prudon, and P. Holliger: Appl. Surf. Sci. 252 (2006) 6478

4) S. Hofmann: Thin Solid Films 398-399 (2001) 336.

5) M. H. Yang and G. G. Goodman: Thin Solid Films 508 (2006) 276.

6) L. Shao, J. Liu, C. Wang, K. B. Ma, J. Zhang, J. Chen, D. Tang, S. Patel, and W.-K. Chu: Nucl. Instrum. Methods Phys. Res., Sect. B 219-220 (2004) 303.

7) A. T. Wu: Physica C 441 (2006) 79

8) D. Donoho: IEEE Trans. Inf. Theory 41 (1995) 613.

9) J.-L. Gauvain and C.-H. Lee: IEEE Trans. Speech Audio Process. 2 (1994) 291.

10) J. N. Flores and S. Young: IEEE Conf. Acoustic Speech and Signal Processing, 1994, p. 829.

11) S. Boll: IEEE Conf. Acoustic Speech and Signal Processing, 1994, p. 2.

12) N. Baboux: Dr. Thesis, INSA of Lyon, France (2001). 